\title{
Raspberry ketone improves diabetic nephropathy in mice by inhibiting streptozotocin-induced oxidative stress and inflammation
}

\section{Xin Wang ( $\triangle$ maritie15843254511@163.com )}

Jilin Agriculture University https://orcid.org/0000-0002-0017-6749

\section{Ying Chang}

Jilin Medical University

\section{Yan Wang}

Institute of Military Veterinary Medicine, Academy of Military Medical Sciences, Zoonosis Prevention and Control Key Laboratory

Yuxin Liu

Yanbian University

\section{Xiyu Hu}

Yanbian University

\section{Yucheng Sun}

Institute of Military Veterinary Medicine, Academy of Military Medical Sciences, Zoonosis Prevention and Control Key Laboratory

\section{Peng Du}

Institute of Military Veterinary Medicine, Academy of Military Medical Sciences, Zoonosis Prevention and Control Key Laboratory

\section{$\mathrm{Na} \mathrm{Xu}$}

Jilin Medical University

Hui Song

Jilin Agricultural University

Wensen Liu

Jilin Agricultural University

\section{Research}

Keywords: Raspberry ketone, Diabetic nephropathy, Oxidative stress, Inflammation

Posted Date: April 23rd, 2020

DOI: https://doi.org/10.21203/rs.3.rs-23174/v1 
License: (c) (i) This work is licensed under a Creative Commons Attribution 4.0 International License. Read Full License 


\section{Abstract \\ Background}

Oxidative stress and inflammation play important roles in the pathogenesis of diabetic nephropathy. Raspberry ketone (RK) is one of the main active substances in raspberry and has a variety of medicinal values. Nevertheless, its protective effect on streptozotocin (STZ)-induced diabetic nephropathy mice has not been reported. In this study, we researched if RK improves oxidative stress and inflammation of STZinduced diabetic nephropathy mice.

\section{Methods}

The model of diabetic nephropathy mice was established by a single injection of STZ $(130 \mathrm{mg} / \mathrm{kg})$. Diabetic nephropathy mice were treated orally with $400 \mathrm{mg} / \mathrm{kg} /$ day raspberry ketone extract to eight weeks. The expression levels of serum creatinine (Scr), urine micro-albumin (mALB), glutathione peroxidase (GSH-Px), superoxide dismutase (SOD), catalase (CAT), malondialdehyde (MDA), tumor necrosis factor alpha (TNF-a), interleukin $1 \beta$ (IL-1 $\beta$ ) and interleukin 6 (IL-6) were measured by Enzymelinked immunosorbent (ELISA). Renal structural changes were investigated by using animal diagnostic ultrasound system.

\section{Results}

RK obviously ameliorated as observed in the visible decrease levels of serum creatinine (Scr), urine microalbumin (mALB). Furthermore, RK improved expression levels of antioxidant enzyme markers (GSH-PX, SOD, CAT) and obviously reduced MDA, a product of lipid peroxidation, pro-inflammatory divisors (TNF-a, IL-6, IL-1 $\beta$ ) in diabetic nephropathy mice. After treatment with RK, we observed that the size of the kidneys and the damage of renal interstitial of mice were obviously improved, as well as the loss of integrity of basement membrane and the renal interstitial edema in diabetic nephropathy mice by using animal diagnostic ultrasound system.

\section{Conclusion}

This study strongly indicates that raspberry ketone provides a protective effect against STZ-induced diabetic nephropathy in improving oxidative stress and inflammation.

\section{Background}

Diabetes mellitus (DM) is currently one of the most severe chronic diseases, which have an important impact on psychological and physiological quality of life. The pathogenesis of DM may be due to acquired insulin deficiency and ineffectiveness of insulin production [1]. On the basis of current 
projections estimate an increase of the prevalence of diabetes by more than $50 \%$ between 2019 and 2045 , leading to around 700 million people suffering from diabetes in the world. Diabetic nephropathy (DN), which are among the most common micro vascular complication in patients with DM, and more than $80 \%$ of end-stage renal diseases (ESRD) are caused by diabetes [1,2]. The number of individuals with diabetic nephropathy accounts for $40 \%$ of patients receiving renal treatment worldwide, and DN is the most causing substantial morbidity and mortality diabetes complication $[3,4]$.

The pathogenesis of diabetic nephropathy is generally believed to be connected with oxidative stress, chronic inflammation and persistent hyperglycemia [3-5]. The evidence indicating that oxidative stress and inflammation play a vital function on STZ-induced diabetic nephropathy $[5,6]$. The existent method geared towards curing diabetic nephropathy hasn't been strongly efficacious, which may be due to the intricacy concerned in pathogenesis of diabetic nephropathy, along with the serious side effects using accompany drugs and therapeutic methods $[1,5]$.

Since oxidative stress caused by disorders of antioxidant enzyme system and inflammation caused by excessive secretion of pro-inflammatory cytokines play an important role in the progress of DM and its complications, officinal plants with potential anti-inflammation and antioxidant effects have given rise enormous attention to the protection and prevention of these complications, consisting of DN. Growing evidence has clearly indicated that ketones in plants have a variety of pharmacological activities, such as anti-diabetes, anti-oxidation and anti-inflammation [6-10]. Raspberry is a plant of the genus raspberry of rosaceae, which has a wide range of medicinal value [9-11]. Raspberry Ketone (RK) is one of the main biological activity components of raspberry, which the functionary mechanism of raspberry ketone of DN is still not fully reported. Consequently, the present experimental study was to research the effect of raspberry ketone on diabetic nephropathy in STZ-induced diabetic mice.

\section{Materials And Methods}

\section{Drugs and reagents}

Raspberry ketone was purchased from Xi'an Herbking Biotechnology Co., Ltd. Glucose assay kit, creatinine (CRE) assay kit, mice urine microalbumin-linked immunoassay kit, superoxide dismutase (SOD) assay kit, glutathione peroxidase (GSH-Px) assay kit, CATalase (CAT) assay kit, and malondialdehyde (MDA) assay kit were purchased from Nanjing Jiancheng Bioengineering Institute, Jiangsu, China. STZ was purchased from Sigma. The high-fat and high-sugar feed was purchased from Changchun Yisi Experimental Animal Technology Co., Ltd. D-glucose was purchased from Biotech Bioengineering (Shanghai) Co., Ltd. IL-6, IL-1 $\beta$ and TNF-a detection kit was purchased from Dakewe Biotech Co., Ltd.

\section{Animal experiments}

Male, 8-week-old C57BL/6J mice, weighing 20-22 g (purchased from Liaoning Changsheng Biotechnology Co., Ltd.). The animals were housed in a standard animal room (temperature: $21-23^{\circ} \mathrm{C}$, 
humidity: 50\%, light: 12: $12 \mathrm{~h}$ light / dark cycle). All the animals received a standard diet and allowed free access to water ad libitum. Mice were acclimatized for one week before starting the experiment. Entire experimental programmes were authorized by the Animal Ethics Committee of Jilin Medical University (2019-Iw001).

\section{Experimental Design}

The mice were fed with ordinary maintenance fodder for a week to help them adapt to the environment. Mice were fed with high-glucose-high-fat diet for four weeks, and injected by once coeliac injection of freshly prepared STZ solution (130 mg/kg). Fasting blood glucose level was measured a week after STZ administration with the aid of glucose assay kit (fasting and freely drinking water for 12 hours). Mice

with fasting plasma glucose level $\geqq 225 \mathrm{mg} \cdot \mathrm{dL}^{-1}$ was used in the experimental research.

In the experiment, mice were randomly divided into 3 groups based on the following treatment scheme (n $=10)$ :

Group I $(n=10)$ Normal control group (Con)

Group II ( $n=10)$ Diabetic nephropathy model group (DN)

Group III ( $(=10)$ Diabetic nephropathy + Raspberry ketone group $(\mathrm{DN}+\mathrm{RK})$

Groups I and II were administered with an equal amount of normal saline daily, group III was administered with raspberry ketone $(400 \mathrm{mg} / \mathrm{kg} /$ day) for a total of 8 weeks. At the end of the experiment, the blood samples were gathered from the venous plexus after the eyeball, and were executed by cervical dislocation. The blood samples were centrifuged at $3000 \mathrm{rpm}$ for $10 \mathrm{~min}$ to obtain the serum and detect biochemical indicators. The kidneys were collected for ultrasonic inspection with each group of mice. Urine was collected from each group for urine-related testing before the mice were executed.

\section{Assessment of Oral Glucose Tolerance Test (OGTT)}

The mice were fasted and freely drank water for 12 hours, and D-glucose ( $2 \mathrm{~g} / \mathrm{kg}$ body weight) was intragastrically administered. The blood glucose levels were detected at $0,30,60,90$, and 120 minutes after gavage administration according to the manufacturer's kit instructions.

\section{Assessment of oxidative stress and antioxidation}

The expressive content of SOD, GSH-Px, MDA and CAT was determined with the help of commercially obtainable assay kit (Nanjing Jiancheng Bioengineering Institute, Jiangsu, China) according to the manufacturer's kit instructions.

\section{Assessment of serum creatinine}

The creatinine was detected in serum with the assistance of a commercially obtainable assay kit (Nanjing Jiancheng Bioengineering Institute, Jiangsu, China) according to the manufacturer's kit instructions. 


\section{Assessment of urine parameters}

Coming to an end of the experiment, the urine at all times of mice was gathered for the detection of urine micro-albumin and urine creatinine. The creatinine clearance was calculated combing urine creatinine with serum creatinine. Creatinine clearance $=$ urinary creatinine $\times 24$ hours urine output $/$ serum creatinine / 2440 min.

\section{Assessment of diabetic serum inflammation}

The expression standard of IL- 6 , TNF- $\alpha$ and IL- $1 \beta$ was assessed by making use of commercially obtainable assay kit (Dakewe Biotech Co., Ltd.) according to the manufacturer's instructions.

\section{Ultrasound assessments}

By the end of the experiment, the changes of kidney size, loss of integrity of the basement membrane and the renal interstitial edema in the different groups were measured by the animal diagnostic ultrasound system.

\section{Statistical Analysis}

The experimental results are indicated as mean \pm SD. Statistical analysis of data was executed making use of GraphPad Prism 8 software (San Diego, California, USA). Statistical dissimilarity significance analysis was determined by Student's t-test or one-way ANOVA. A p-value $<0.05$ was considered statistically significant.

\section{Results}

\section{Effect of raspberry ketone on renal hypertrophy}

We investigated the effects of raspberry ketone on renal hypertrophy in diabetic nephropathy mice. Experimental studies have found that the kidney weight / body weight ratio and kidney cross-sectional area of diabetic nephropathy model mice are increased compared to the normal control group. This indicates that the kidneys of diabetic mice are hypertrophic. After 8 weeks of raspberry ketone treatment, the kidney weight / body weight ratio and kidney cross-sectional area of mice were significantly reduced compared with the model group. (Fig. 1)

\section{Effect of raspberry ketone on renal injury}

We investigated the effects of raspberry ketone on creatinine clearance and urine microalbumin in diabetic nephropathy mice. Compared with the normal control group, the expressions of urine microalbumin and serum creatinine in diabetic nephropathy model group mice were significantly increased, and the urine creatinine ( $\mathrm{UCr}$ ) and creatinine clearance rates (Ccr) were significantly reduced. However, after 8 weeks of treatment with raspberry ketones, creatinine clearance and urinary microalbumin were significantly improved in diabetic nephropathy mice. (Fig. 2) 


\section{Effect of raspberry ketone on glucose tolerance}

In order to evaluate the impact of raspberry ketone on blood glucose in mice, the OGTT levels and the oral glucose tolerance test-area under the curve (OGTT-AUC) are shown in Fig. 3. The level of OGTT was an apparent rise in diabetic nephropathy model group in comparison to the normal mice. However, the level of OGTT was transparently decreased in raspberry ketone group in comparison to the diabetic nephropathy model group. In addition, the area under curve analysis also indicated that the blood glucose level was markedly reduced after treatment with raspberry ketone.

\section{Effect of raspberry ketone on renal oxidative stress}

The effect of raspberry ketone treatment on antioxidant enzyme biological activities and oxidative stress indicators were indicated in Fig. 4. In our experiment, the level of MDA in diabetic nephropathy model control mice was markedly higher compared with the normal control mice. Furthermore, the biological activities of the antioxidant enzymes, namely, SOD, CAT and GSH-PX in diabetic nephropathy model control mice were significantly reduced compared with normal control mice. However, treatment with raspberry ketone made MDA significantly reduced, markedly strengthening the biological activities of GSH, SOD and CAT in comparison to diabetic nephropathy model group. It has been proved that raspberry ketone can improve the oxidative stress in diabetic nephropathy mice. (Fig. 4)

\section{Effect of raspberry ketone on inflammatory factors}

The effect of raspberry ketone on pro-inflammatory cytokines is indicated in Fig. $5 \mathrm{~A}-\mathrm{C}$. The levels of proinflammatory cytokines (TNF-a, IL-6, IL-1 $\beta$ ) were markedly higher in diabetic nephropathy model control mice. However, the level of pro-inflammatory cytokines in raspberry ketone group was significantly lowered than diabetic nephropathy model control mice. It was proved that raspberry ketone can improve the inflammation of mice with diabetic nephropathy. (Fig. 5)

\section{Effect of raspberry ketone on renal changes}

To assess whether raspberry ketones could improve the renal pathology of diabetic nephropathy, we used an animal diagnostic ultrasound system at 8 weeks of treatment to observe changes in kidney size and mouse kidney interstitial changes. The results showed that kidney hypertrophy, loss of basement membrane integrity and renal interstitial edema were observed in the model group of mice. After 8 weeks of treatment with raspberry ketone, the kidney structure of the mice improved significantly. (Fig. 6)

\section{Discussion}

Diabetic nephropathy is a grievous public health problem, which considered to among the grievous lifethreatening complications of diabetes mellitus, and among the most important cause of end-stage renal disease (ESRD) [12]. Diabetic nephropathy occurs in morbidity reach up to $30 \sim 40 \%$ of diabetes 
complications. Diabetic nephropathy is featured by augmented proteinuria and glomerular filtration dysfunction in the clinical. There are some significant pathological changes in the shape and function of the renal, for example, renal microvasculature disruption, glomerular capillaries, tubular interstitial damage renal hypertrophy and expansion of the extracellular matrix [13]. Diabetic nephropathy brings great economic burden to people, and the prognosis of patients is poor, and the morbidity and mortality are high, which has caused widespread concern and research. Although treatments for diabetic nephropathy continue to develop, morbidity and mortality appear also to be increasing [14]. Therefore, seeking for more efficacious selectable treatment from officinal herbs in reducing the incidence and/or mortality of diabetic nephropathy is ongoing.

Raspberry Ketone is a well-known traditional Chinese medicine. In recent years, it has become a research hotspot because of its various biological activities such as anti-hyperglycemic, antioxidant, antiinflammatory and neuroprotective [9-11]. The plants are rich in ketone compounds and are a good antidiabetic drug, which can further explore the effect of the plant extracts on diabetic complications such as renal disease. The current research, consequently, appraised the protective effect of Chinese herbal extracts for DN in STZ-induced diabetic mice.

Oxidative stress plays an important role that has been incriminated in the nosogenesis of DN [15]. Excessive blood glucose is given rise to overproduction of oxidative stress, which has been intimately related to the occurrence and progress of DN [16-20]. Raising blood glucose is given rise to the overproduction of oxidative stress in the renal, which is given rise to the apoptosis of proximal tubular epithelial cells and mechanical cells and the depletion of phagocytes, which is the progression of diabetic nephropathy A phase is set $[24,25]$. Raising biological activities of antioxidant enzymes alleviate oxidative stress, and foregoing research studies have shown that some antioxidant enzymes are decreased and lipid peroxidations are raised in DN [18]. Previous research has discovered that malondialdehyde with lipid peroxidation production was markedly raised, while the biological activity of SOD, GSH-PX and CAT was in diabetic nephropathy animals with a downturn $[17,26]$. The research discovered that after treatment with raspberry ketone, diabetic mice markedly raised the biological activities of the internal SOD, GSH-PX and CAT, and concurrently given rise to a descendant in the malondialdehyde of lipid peroxidation production level. It validates that raspberry ketone has a beneficial antioxidant effect, while the mechanisms needed further investigation.

The excessive secretion of the pro-inflammatory cytokine has been put forward to bring into play an essential function on the occurrence and progression of DN [21-23]. Preceding experimental research has found that the production level of pro-inflammatory cytokines, for example, IL-1 $\beta$ and IL-6, TNF-a is increased in diabetic nephropathy $[27,28]$. Stimulating an increase in the expression of various proinflammatory cytokines goes through the stimulation of NF-KB pathway when the organism produces oxidative stress, thus damaging the renal tissue [29]. Foregone experimental studies have shown that proinflammatory cytokines affected renal function and renal damage in a few mechanistic pathways [20, 30, 34]. Furthermore, the excessive release of pro-inflammatory cytokines in DN may give rise to the infiltration of macrophages, which successively injures the glomerular and causes excessive the level of 
urine micro-albumin $[27,31,32]$. Our experimental results showed that the production of TNF-a, IL-6 and IL-1 $\beta$ increased in diabetic nephropathy model control mice, which were markedly declined by raspberry ketone treatment. These results indicated that raspberry ketone may improve the renal function by reducing the expression of several pro-inflammatory cytokines, while the mechanisms needed further investigation.

The abnormal excretion of creatinine and urine micro-albumin is regarded as an important clinical pathological sign of diabetic nephropathy $[35,36]$. Injuries to the renal in STZ-induced diabetic nephropathy animals are obvious with the increase in serum creatinine and the decrease in urine creatinine, which are suggestive of the abatement in creatinine clearance. Our experiments discover that raspberry ketone therapy reduced serum creatinine and raised urine creatinine levels in diabetic mice, indicating an enhancement in the renal functions of diabetic animals of the raspberry ketone therapy.

In our experimental animal model with revulsive diabetic nephropathy, animal ultrasound analysis of diabetic mice indicated noteworthy that the renal lesion was serious in diabetic nephropathy model group but the kidneys of the treated mice were similar in size to normal mice and the loss of integrity of basement membrane and was improved. Renal interstitial edema also was improved. We prove that raspberry ketone improved a few representative changes in diabetic nephropathy, namely, renal inflammation, renal hypertrophy, loss of integrity of renal basement membrane and renal interstitial edema, while the specific pathological analysis needed further investigation.

\section{Conclusion}

In the present study, our experimental results showed that raspberry ketone ameliorated kidney damage caused by STZ-induced diabetic mice in the way of improving antioxidant enzymes biological activities and reducing secretion of pro-inflammatory cytokines, indicating that raspberry ketone might have profitable curative effect on the prophylaxis and medication of the diabetic nephropathy. Nevertheless, further investigations are necessary prior to clinical application.

\section{Abbreviations}

\section{RK}

Raspberry ketone

STZ

streptozotocin

Scr

serum creatinine

MALB

urine micro-albumin

GSH-PX

glutathione peroxidase 
SOD

superoxide dismutase

CAT

catalase

MDA

malondialdehyde

TNF-a

Tumor necrosis factor alpha

IL-1 $\beta$

Interleukin-1 $\beta$

IL-6

Interleukin-6

ELISA

Enzyme linked immunosorbent assay

DM

Diabetes mellitus

DN

Diabetic nephropathy

ESRD

end-stage renal diseases

CRE

creatinine

OGTT

Oral Glucose Tolerance Test

$\mathrm{UCr}$

urine creatinine

Ccr

creatinine clearance rates

Con

Normal control group

DN

Diabetic nephropathy model group

DN + RK

Diabetic nephropathy + Raspberry ketone group

OGTT-AUC

oral glucose tolerance test-area under the curve

\section{Declarations}

Ethics approval and consent to participate 
Not applicable.

\section{Consent for publication}

Not applicable.

\section{Availability of data and materials}

Not applicable.

\section{Competing interests}

The authors declare that they have no competing interests.

\section{Funding}

This research work was financially supported by the grant from the Project Agreement for Science \& Technology Development, Jilin Province (No. 20190304027YY, 20180201033YY)

\section{Authors' contributions}

XW and YC performed the study. XW designed and analyzed the data and revised the manuscript. NX and W-SL organized and supervised the study. All authors read and approved the final manuscript.

\section{Acknowledgements}

Not applicable.

\section{Author information}

\section{Affiliations}

School of Life Sciences Jilin Agricultural University, Changchun, 130000, PR China

Xin Wang, Hui Song \& Wensen Liu

Institute of Military Veterinary Medicine, Academy of Military Medical Sciences, Zoonosis Prevention and Control Key Laboratory, Changchun, 130122, PR China

Yan Wang, Yucheng Sun \& Peng Du

Jilin Medical University, Jilin, 132013, PR China

Ying Chang \& $\mathrm{Na} \mathrm{Xu}$

Medical College, Yanbian University, Yanji, 133002, PR China 
Yuxin Liu \& Xiyu Hu

\section{Corresponding authors}

Correspondence to Na Xu, Hui Song or Wensen Liu.

\section{References}

1. Gross JL, de Azevedo MJ, Silveiro SP, Canani LH, Caramori ML, Zelmanovitz T. Diabetic nephropathy: diagnosis, prevention, and treatment. Diabetes Care. 2005 Jan;28(1):164-76.

2. Pourfarjam Y, et al. Effect of Cichorium intybus L. seed extract on renal parameters in experimentally induced early and late diabetes type 2 in rats. Ren Fail. 2017;39(1):211-21.

3. Rehman K, Akash MSH. Mechanism of Generation of Oxidative Stress and Pathophysiology of Type 2 Diabetes Mellitus: How Are They Interlinked? J Cell Biochem. 2017;118(11):3577-85.

4. Ighodaro OM. Molecular pathways associated with oxidative stress in diabetes mellitus. Biomedicine\& Pharmacotherapy. 2018;108:656-62.

5. Gnudi L, Coward RJM, Long DA. Diabetic Nephropathy: Perspective on Novel Molecular Mechanisms. Trends Endocrinol Metab. 2016;27(11):820-30.

6. Lu YX, et al. Antidiabetic effects of total flavonoids from Litsea Coreana leve on fat-fed, streptozotocin-induced type 2 diabetic rats. Am J Chin Med. 2010;38(4):713-25.

7. Khazaei M, et al. Effects of Resveratrol on Receptor for Advanced Glycation End Products (RAGE) Expression and Oxidative Stress in the Liver of Rats with Type 2 Diabetes. Phytother Res. 2016;30(1):66-71.

8. Ren $B$, et al. Apigenin and naringenin regulate glucose and lipid metabolism, and ameliorate vascular dysfunction in type 2 diabetic rats. Eur J Pharmacol. 2016;773:13-23.

9. Mohamed HE, et al. Raspberry ketone preserved cholinergic activity and antioxidant defense in obesity induced Alzheimer disease in rats. Biomed Pharmacother. 2018;107:1166-74.

10. Attia RT, Abdel-Mottaleb Y, Abdallah DM, El-Abhar HS, El-Maraghy NN. Raspberry ketone and Garcinia Cambogia rebalanced disrupted insulin resistance and leptin signaling in rats fed high fat fructose diet. Biomed Pharmacother. 2019 Feb;110:500-9.

11. Khan V, et al. Suppression of isoproterenol-induced cardiotoxicity in rats by raspberry ketone via activation of peroxisome proliferator activated receptor-a. Eur J Pharmacol. 2019;842:157-66.

12. Tesch GH. Diabetic nephropathy-is this an immune disorder? Clin Sci. 2017;131(16):2183-99.

13. Jha JC, et al. Diabetes and Kidney Disease: Role of Oxidative Stress. Antioxid Redox Signal. 2016;25(12):657-84.

14. Ilyas Z, Chaiban JT, Krikorian A. Novel insights into the pathophysiology and clinical aspects of diabetic nephropathy. Reviews in Endocrine Metabolic Disorders. 2017;18(1):21-8. 
15. Olatunji OJ, Chen H, Zhou Y. Lycium chinense leaves extract ameliorates diabetic nephropathy by suppressing hyperglycemia mediated renal oxidative stress and inflammation. Biomed Pharmacother. 2018;102:1145-51.

16. Khanra R, Dewanjee S, K Dua T, Sahu R, Gangopadhyay M, De Feo V, Zia-Ul-Haq M. Abroma augusta L. (Malvaceae) leaf extract attenuates diabetes induced nephropathy and cardiomyopathy via inhibition of oxidative stress and inflammatory response. J Transl Med. 2015 Jan;16:13:6.

17. Yiu WH, et al. Kallistatin protects against diabetic nephropathy in $\mathrm{db} / \mathrm{db}$ mice by suppressing AGERAGE-induced oxidative stress. Kidney Int. 2016;89(2):386-98.

18. Hong YA, et al. Extracellular Superoxide Dismutase Attenuates Renal Oxidative Stress Through the Activation of Adenosine Monophosphate-Activated Protein Kinase in Diabetic Nephropathy. Antioxid Redox Signal. 2018;28(17):1543-61.

19. Coughlan MT, Sharma K. Challenging the dogma of mitochondrial reactive oxygen species overproduction in diabetic kidney disease. Kidney Int. 2016;90(2):272-9.

20. Fakhruddin S, Alanazi W. and K.E. Jackson. Diabetes-Induced Reactive Oxygen Species: Mechanism of Their Generation and Role in Renal Injury. Journal of Diabetes Research. 2017;2017:1-30.

21. Liang G, et al. Fibroblast growth factor 1 ameliorates diabetic nephropathy by an anti-inflammatory mechanism. Kidney Int. 2018;93(1):95-109.

22. Han-Qing W, Sha-Sha W, Kuok C, Xiao-Lan QW. Ch. Umbelliferone ameliorates renal function in diabetic nephropathy rats through regulating inflammation and TLR_NF-KB pathway. Chin J Nat Med. 2019;17(5):0346-54.

23. Gangadharan Komala $M$, et al. Saxagliptin reduces renal tubulointerstitial inflammation, hypertrophy and fibrosis in diabetes. Nephrology (Carlton). 2016;21(5):423-31.

24. Falkevall A, et al. Reducing VEGF-B Signaling Ameliorates Renal Lipotoxicity and Protects against Diabetic Kidney Disease. Cell Metab. 2017;25(3):713-26.

25. Ying $C$, et al. Bamboo leaf extract ameliorates diabetic nephropathy through activating the AKT signaling pathway in rats. Int J Biol Macromol. 2017;105:1587-94.

26. Kim BH, et al. Protective Effects of Curcumin on Renal Oxidative Stress and Lipid Metabolism in a Rat Model of Type 2 Diabetic Nephropathy. Yonsei Med J. 2016;57(3):664-73.

27. Pandey A, et al. H2AK119 monoubiquitination regulates Angiotensin II receptor mediated macrophage infiltration and renal fibrosis in type 2 diabetic rats. Biochimie. 2016;131:68-76.

28. Li J, et al. Inhibitor of IGF1 receptor alleviates the inflammation process in the diabetic kidney mouse model without activating SOCS2. Drug Design, Development and Therapy, 2018. Volume 12:28872896.

29. Xie Z, et al. Carnosic acid improves diabetic nephropathy by activating Nrf2/ARE and inhibition of NF-kB pathway. Phytomedicine. 2018;47:161-73.

30. Xin R, et al. Apocynin inhibited NLRP3/XIAP signalling to alleviate renal fibrotic injury in rat diabetic nephropathy. Biomed Pharmacother. 2018;106:1325-31. 
31. Klessens CQF, et al. Macrophages in diabetic nephropathy in patients with type 2 diabetes. Nephrol Dial Transplant. 2017;32(8):1322-9.

32. Zhang M-Z, et al. Lysophosphatidic Acid Receptor Antagonism Protects against Diabetic Nephropathy in a Type 2 Diabetic Model. J Am Soc Nephrol. 2017;28(11):3300-11.

33. Wada J, Makino H. Innate immunity in diabetes and diabetic nephropathy. Nat Rev Nephrol. 2016;12(1):13-26.

34. Rops ALWMM, et al. Interleukin- 6 is essential for glomerular immunoglobulin A deposition and the development of renal pathology in Cd37-deficient mice. Kidney Int. 2018;93(6):1356-66.

35. Tanaka S, et al. Sodium-glucose cotransporter 2 inhibition normalizes glucose metabolism and suppresses oxidative stress in the kidneys of diabetic mice. Kidney Int. 2018;94(5):912-25.

36. Boonloh K, et al. Rice bran protein hydrolysates attenuate diabetic nephropathy in diabetic animal model. Eur J Nutr. 2016;57(2):761-72.

\section{Figures}

A

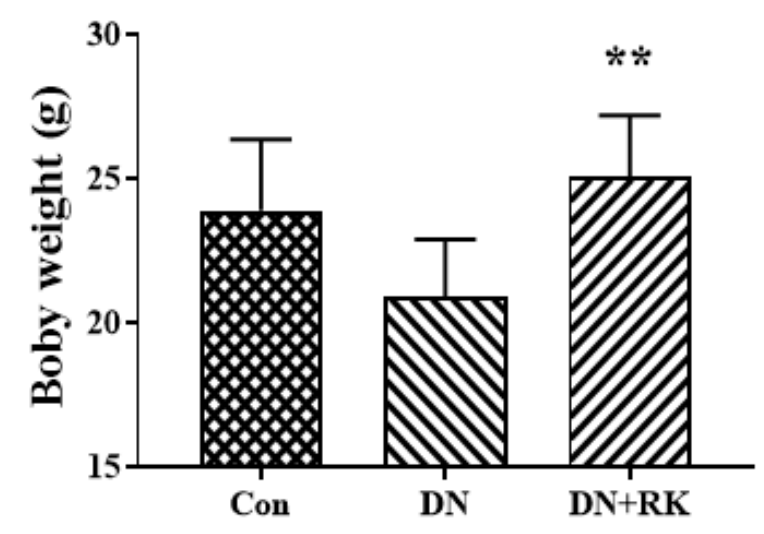

C

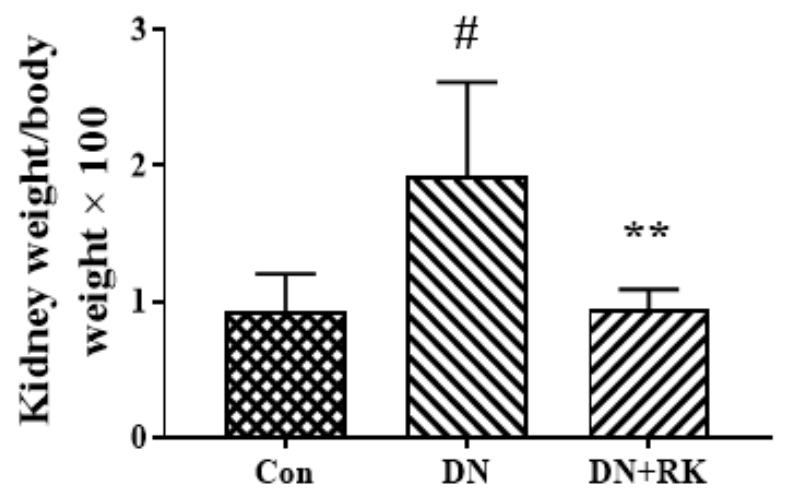

B

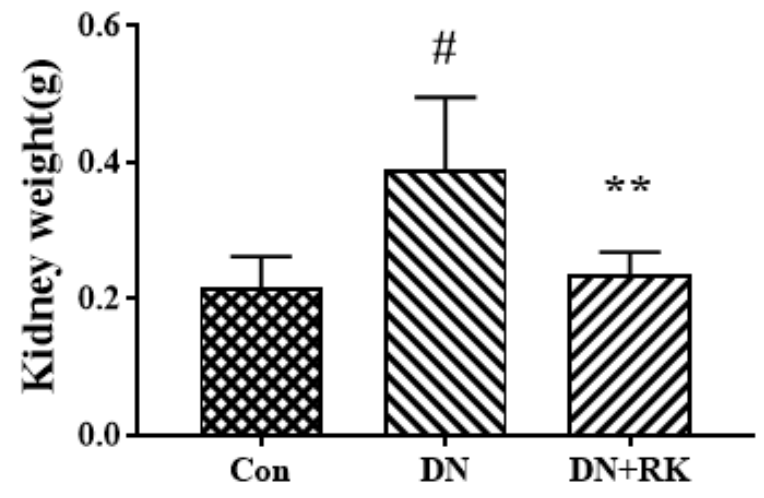

D

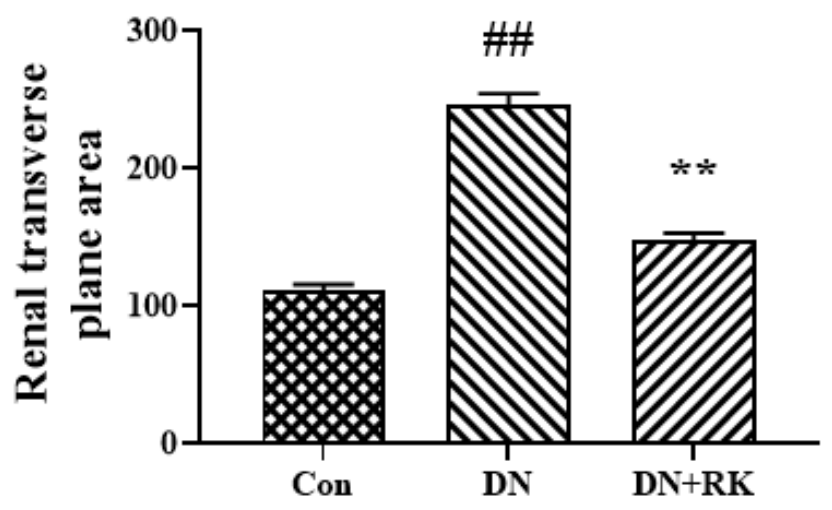

Figure 1 
Effect of raspberry ketone on kidney hypertrophy in STZ-induced diabetic nephropathy mice. (A): Body weight; (B): Kidney weight; (C): Kidney weight/Body weight $\times 100$; (D) Renal transverse plane area. Values are indicated as mean $\pm S D(n=10)$. $\# p<0.05$ and $\# \# p<0.01$ as indicated to the normal control group. $* * p<0.01$ when indicated to diabetic nephropathy model group.

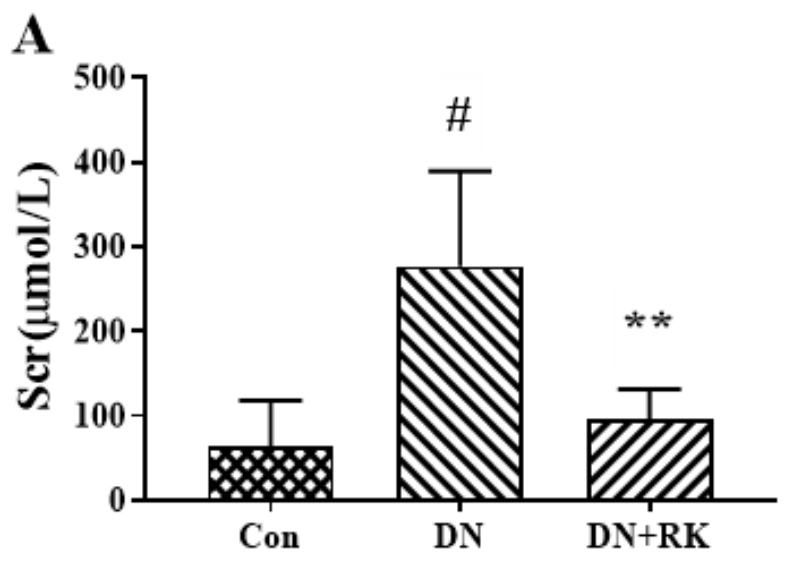

C

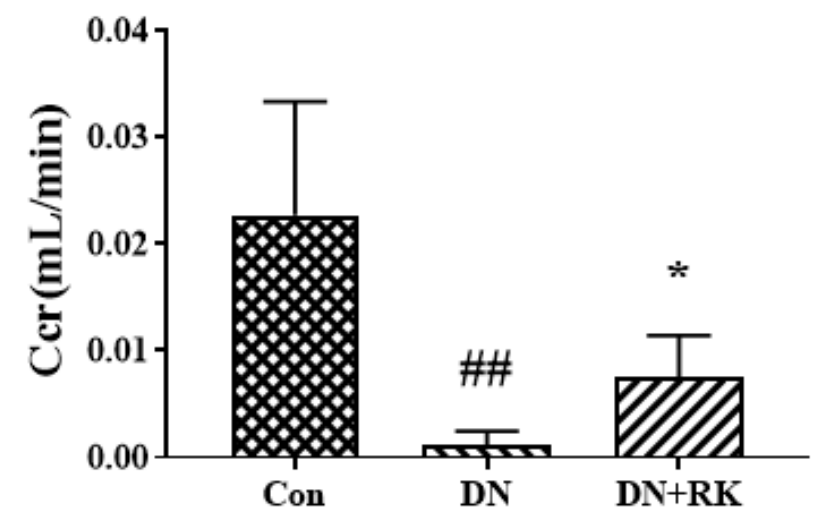

B

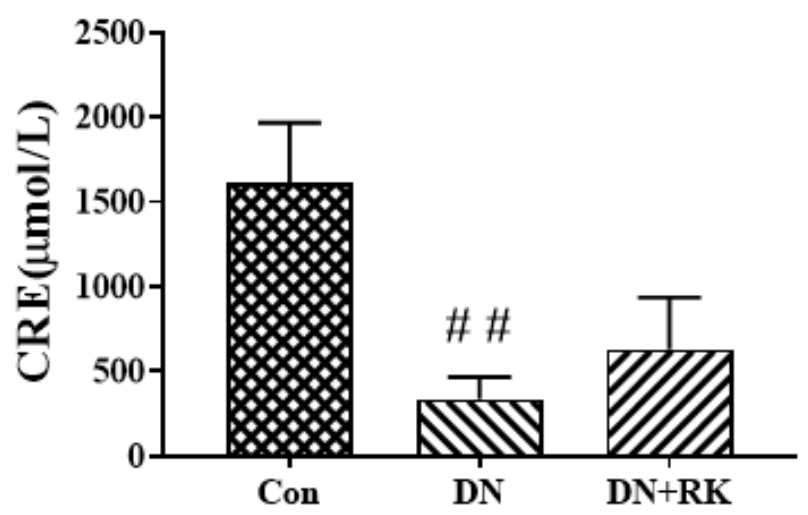

D

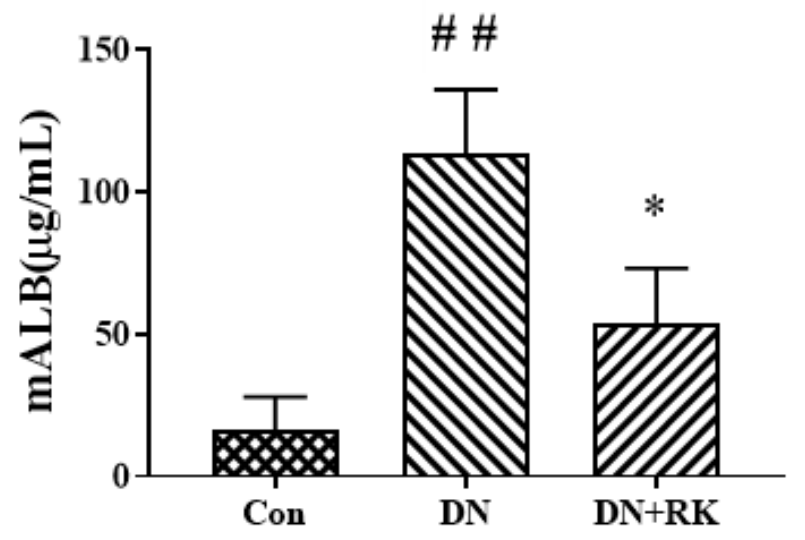

Figure 2

Effect of raspberry ketone on serum biochemical parameters (A) Scr (B) UCr (C) Ccr (D) mALB in STZinduced diabetic nephropathy mice. Values are indicated as mean $\pm S D(n=10)$. \#p<0.05 and \#\#p<0.01 as indicated to normal control group. ${ }^{*} \mathrm{p}<0.05$ and ${ }^{* *} \mathrm{P}<0.01$ when indicated to diabetic nephropathy model group. 

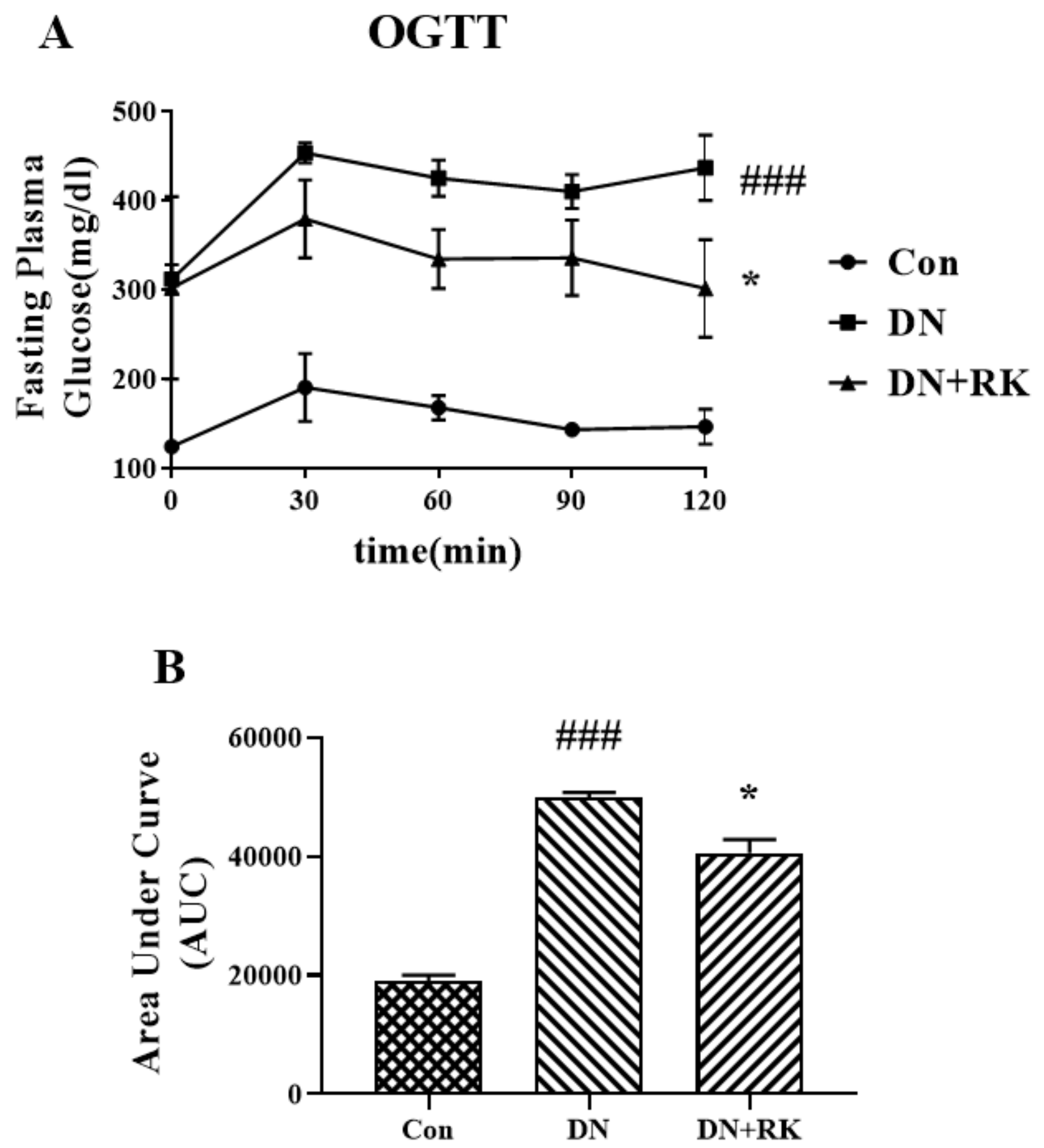

Figure 3

Effect of raspberry ketone on glucose tolerance in diabetic mice. (A) OGTT (B) AUC. Values are indicated as mean $\pm S D(n=10)$. \#\#\#p<0.001 as indicated to normal control group. ${ }^{*}<<0.05$ when indicated to diabetic nephropathy model group. 
A

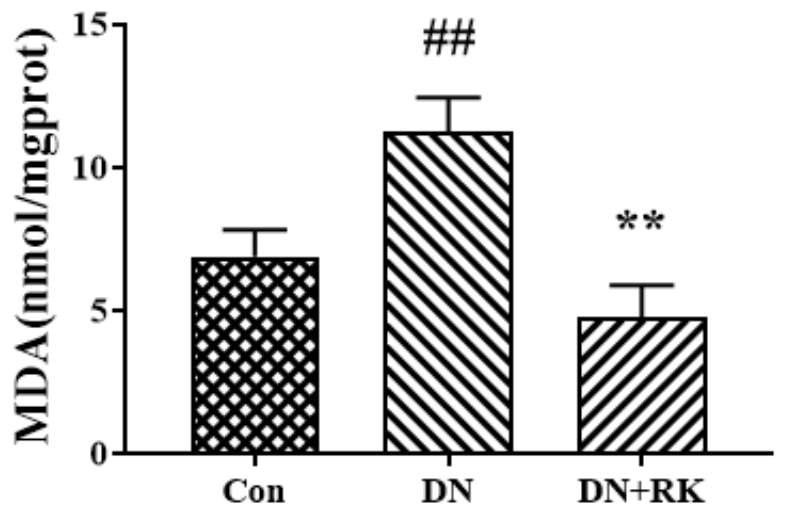

C

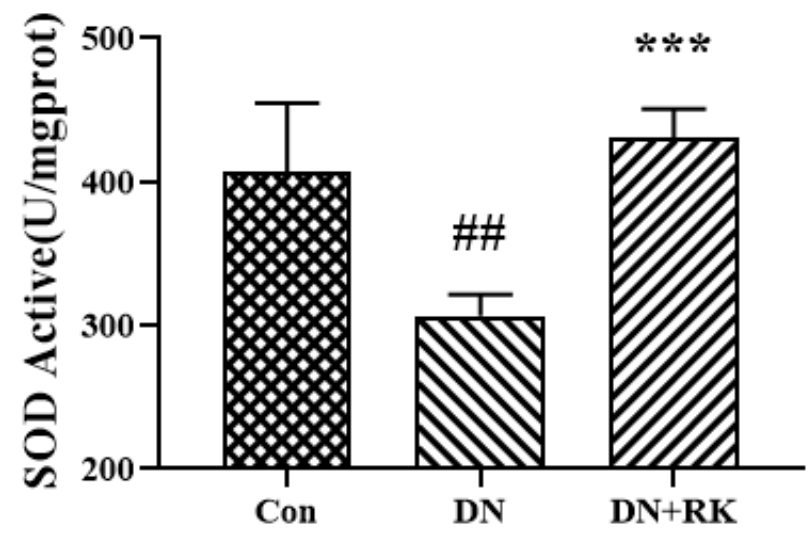

B

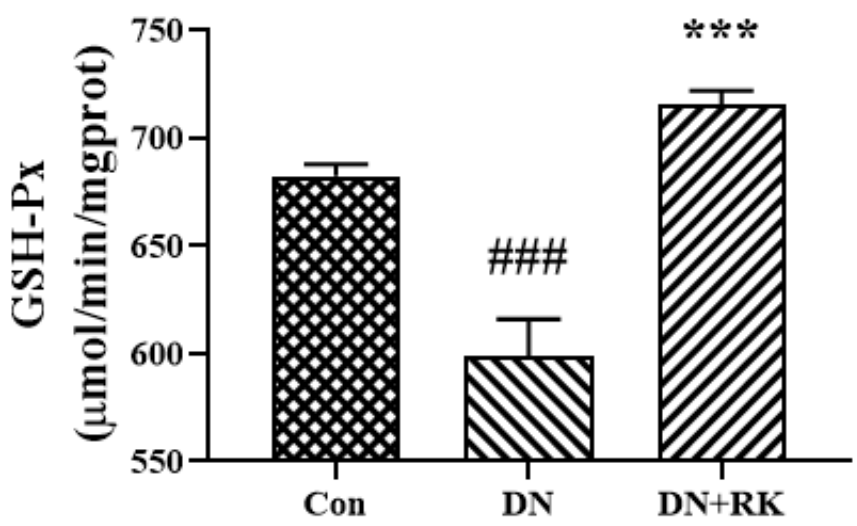

D

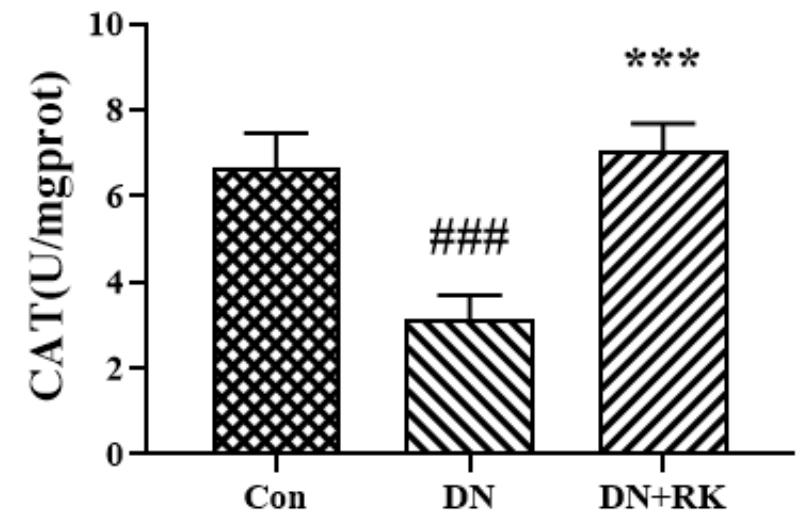

Figure 4

Effect of raspberry ketone on serum the antioxidant enzymes and oxidative stress indicators (A) MDA (B) GSH-Px (C) SOD (D) CAT biological activities in STZ-induced diabetic nephropathy mice. Values are indicated as mean $\pm S D(n=10)$. \#\#p<0.01 and \#\#\#p<0.001 as indicated to normal control group. ${ }^{*} p<0.05$ and ${ }^{* \star *} P<0.001$ when indicated to diabetic nephropathy model group. 

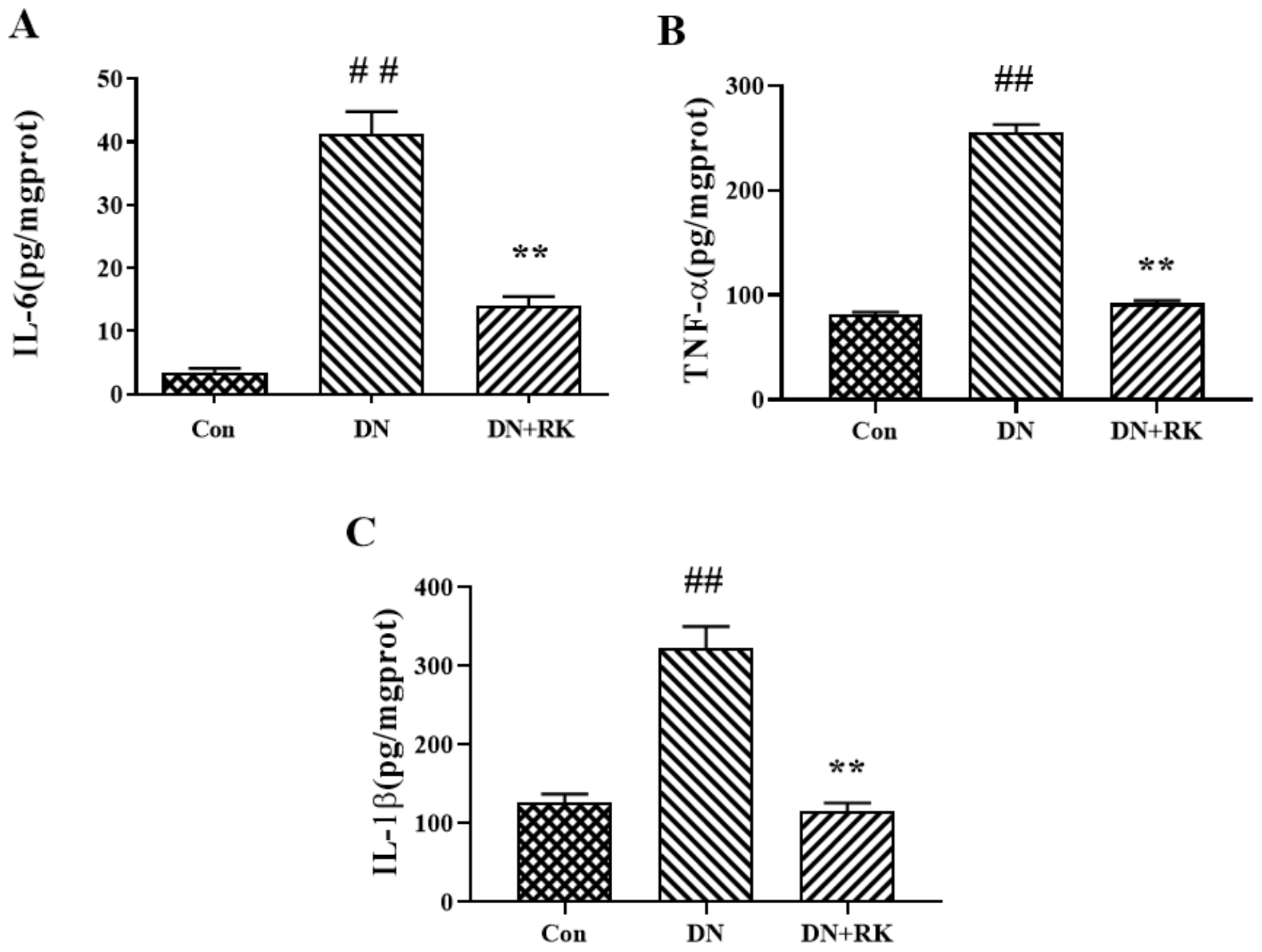

Figure 5

Effect of raspberry ketone on serum pro-inflammatory cytokines (A) IL-6 (B) TNF- $a$ (C) IL-1 $\beta$ in STZinduced diabetic nephropathy mice. Values are indicated as mean $\pm \operatorname{SD}(n=10)$. \#\#p<0.01 as indicated to normal control group. ${ }^{\star *} \mathrm{P}<0.01$ when indicated to diabetic nephropathy model group. 

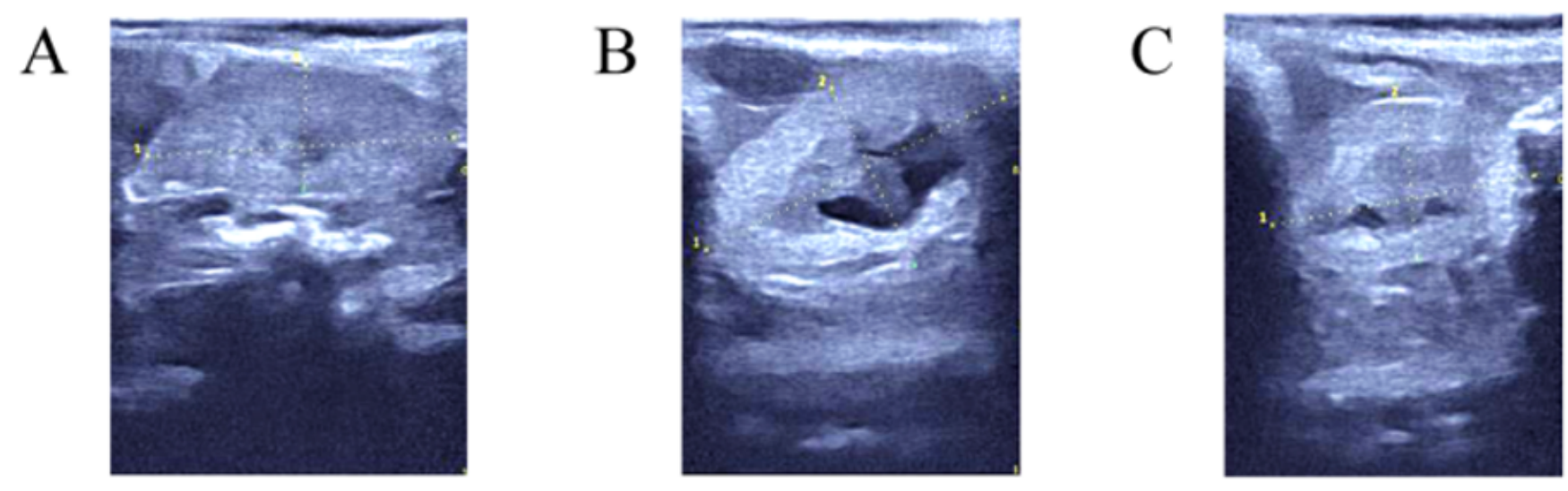

\section{Figure 6}

Ultrasonic images of representative pathological structure of mice kidney. (A): Normal control group (Con); (B): Diabetic nephropathy model group (DN); (C): Diabetic nephropathy +Raspberry ketone group $(\mathrm{DN}+\mathrm{RK})$. 